\section{Antipsychotic drugs and metabolic syndrome - can we prevent it?}

\author{
Drogas antipsicóticas e \\ a síndrome metabólica - \\ podemos evitá-la?
}

The use of first-and second-generation antipsychotic drugs led to an unequivocal improvement in the medical treatment of serious mental illnesses, such as schizophrenia. However, treatment with these drugs is associated with important side effects such as obesity, impaired glucose metabolism, dyslipidemia, arterial hypertension, all of them being phenotypes related with insulin resistance and collectively termed metabolic syndrome (MS). This syndrome, which affects one-quarter of the American population, may have serious consequences for cardiovascular disease (CVD) and diabetes development. Furthermore, epidemiological studies demonstrated that the MS is highly prevalent among treated patients with schizophrenia. ${ }^{1}$ The reasons for this association between antipsychotics drugs and MS remains unclear but the alteration in food intake, reduction in physical activity and direct or indirect effects in mechanisms of insulin action and/or secretion may be implicated. The study of Assunção et al. demonstrated that the use of olanzapine with nizatidine was not effective in controlling weight gain in patients with schizophrenia. ${ }^{2}$ Patients had a mean weight gain of $\sim 12 \%$ from baseline randomization to endpoint after 12 weeks. These results reinforce the concept that behavioral modification, such as physical activity, which promotes a tremendous effect in the protection against metabolic disturbances (e.g. diabetes and obesity), probably should be the treatment of choice for these patients. Indeed, all components of the metabolic syndrome are improved by even modest amounts of weight loss achieved with diet and exercise.

The paper of Leitão-Azevedo et al. found an increased rate of dyslipidemia in schizophrenic patients using some new generation antipsychotics, mainly lower HDL-cholesterol levels. ${ }^{3}$ Furthermore, this study found a very high prevalence of prediabetes ( $40 \%)$ by using the last American Diabetes Association cut-off for the diagnosis of diabetes and prediabetes (ADA-2003). However, the proposed revised diagnostic criteria are not free of criticism. The adoption of these criteria will lead to a dramatic increase in the prevalence of impaired fasting glucose. It is true that these new criteria can identify subjects with real risk of developing diabetes in the future but, at the same time, lead to a pandemic of prediabetes. About $25-40 \%$ of the adults around the world will have prediabetes. ${ }^{4}$ This seriously challenges whether the existing intervention strategies to prevent diabetes (physical activity, weight loss, and the use of metformin) are applicable to the new category of individuals with impaired fasting glycemia.

Because of the close associations between obesity, diabetes, and dyslipidemia with CVD, a recent Consensus was made on Antipsychotic Drugs and Obesity and Diabetes, with the participation of representatives of the American Diabetes Association and American Psychiatric Association among others. ${ }^{5}$ The panel recommended that baseline screening measures should be obtained before, or as soon as clinically feasible after, the initiation of any antipsychotic medication. These include: Personal and family history of obesity and diabetes, dyslipidemia, 
hypertension, or cardiovascular disease. Weight and height (so that Body Mass Index can be calculated; waist circumference), blood pressure, fasting plasma glucose and lipid profile. Fasting plasma glucose, lipid levels, and blood pressure should also be assessed 3 months after initiation of antipsychotic medications. Thereafter, blood pressure and plasma glucose values should be obtained annually or more frequently in those who have a higher baseline risk for the development of diabetes or hypertension. In those with a normal lipid profile, testing should be performed at 5-year intervals or more frequently if clinically indicated.

In conclusion, collaboration between the psychiatric and diabetology or endocrinologist teams is essential to minimize the risk of diabetes, obesity and other co-morbidities in patients taking second-generation antipsychotic medications and for effective management when it develops. This population, which already has a high burden of physical morbidity and excess mortality, should be peremptorily closely monitored, offered appropriate screening and, where necessary, treatment and perhaps prevention of these conditions. This collaboration will help minimize the already high risk of cardiovascular disease in individuals with schizophrenia and other major mental health disorders. The question if we can prevent MS in patients using antipsychotic drugs remains unanswered but a large body of data suggests that weight loss achieved with diet and exercise could be the most effective way to promote wealth quality, protecting against these metabolic dysfunctions.

André $\mathrm{F}$ Reis Laboratory of Molecular Endocrinology, Escola Paulista de Medicina, Universidade Federal de São Paulo, São Paulo (SP), Brazil Diabetes Center, Fleury Institute, São Paulo (SP), Brazil

\section{References}

1. Mackin P, Watkinson HM, Young AH. Prevalence of obesity, glucose homeostasis disorders and metabolic syndrome in psychiatric patients taking typical or atypical antipsychotic drugs: a crosssectional study. Diabetologia. 2005;48(2):215-21.

2. Assunção SS, Ruschel SI, Rosa LC, Campos JA, Alves MJ, Bracco OL. Weight gain management in patients with schizophrenia during treatment with olanzapine in association with nizatidine. Rev Bras Psiquiatr. 2006;28(4):270-6.

3. Leitão-Azevedo C, Guimaraes LR, Belmonte de Abreu MG, Gama CS, Lobato MI, Belmonte-de-Abreu PS. Increased dyslipidemia in schizophrenic outpatients using new generation antipsyhcotics. Rev Bras Psiquiatr. 2006;28(4):301-4.

4. Borch-Johnsen K, Colagiuri S, Balkau B, Glumer C, Carstensen B, Ramachandran A, Dong Y, Gao W. Creating a pandemic of prediabetes: the proposed new diagnostic criteria for impaired fasting glycaemia. Diabetologia. 2004;47(8):1396-402.

5. American Diabetes Association; American Psychiatric Association; American Association of Clinical Endocrinologists; North American Association for the Study of Obesity. Consensus Development Conference on Antipsychotic Drugs and Obesity and Diabetes. Diabetes Care. 2004;27(2):596-601. 